\title{
Analyse comparative du seston estival de 9 lacs du Jura français ${ }^{1}$
}

\author{
C. Martin $^{2}$
}

Mots clés : Lacs, seston, matière organique particulaire, fonctionnement.

Les premières données relatives au seston des lacs du Jura sont présentées. 9 lacs ont fait l'objet de prélèvements en fin de période estivale, par pompage intégré à la verticale du point de profondeur maximale. La matière en suspension totale (MEST) varie selon les systèmes de $0,89 \mathrm{mg} / 1$ à $5,51 \mathrm{mg} / \mathrm{l}$.

Trois cas de figure se distinguent dans le seston des lacs étudiés : seston à prolifération planctonique, secton à trypton dominant, seston « équilibré ». Sur la moyenne des échantillons, la fraction détritique représente $30 \%$ du nombre des particules en suspension.

L'analyse microparticulaire a conduit à l'identification et à la définition de diverses catégories de particules organiques regroupées en deux grands ensembles : les éléments figurés et les associations organo-minérales composites. Dans les échantillons à prolifération planctonique ou à trypton dominant, les macrophytoclastes et les associations organominérales composites présentent leurs abondances maximales.

Une interprétation est proposée relative à la formation des associations organo-minérales en rapport avec différents facteurs écologiques : importance de la frange littorale, couple oxygène/température, prolifération planctonique. Les résultats sont discutés en relation avec le mode de fonctionnement des systèmes.

\section{Comparative study of summer seston from nine lakes of the French Jura}

Keywords : Lake, seston, particulate organic matter, dynamic.

First investigations on seston from lakes of the French Jura are presented. Nine lakes were analysed after having been sampled at the end of summer by vertical pumping at the deepest point of the lakes. Total Suspended Matter (TSM) concentrations vary from $0,89 \mathrm{mg} / 1$ to $5,51 \mathrm{mg} / 1$.

Throughout the results, three types of lakes can be distinguished : planktonic proliferation lakes, lakes showing dominating trypton, well-balanced lakes. Detrital fraction represents an average of $30 \%$ of Total Suspended Particle Number.

A microscopy study produced a classification with two orders of organic particles : detritus, and Heterogeneous Mineral and Organic Compounds. Macrophyte detritus and Heterogeneous Mineral and Organic Compounds, are dominant in seston of lakes where planktonic blooms take place or in lakes where trypton is numerically highest.

A mode of production of Heterogeneous Mineral and Organic Compounds is proposed and discussed in relation to some ecological factors : littoral zone development, oxygen/temperature effects, and planktonic blooms. A general interpretation of the results in relation to Particulate Organic Matter transfer into the Limnological Ecosystem is also given.

1. Recherches faisant partie d'un programme soutenu par le Conseil Régional de France-Comté et l'Agence de l'Eau Rhône-MéditerranéeCorse.

2. Laboratoire d'Hydrobiologie-Hydroécologie, Institut des Sciences et Techniques de l'Environnement, Université de Franche-Comté, place Leclerc, 25030 Besançon Cedex, France.

Texte d'une communication effectuée dans le cadre du Colloque - Limnologie appliquée et application de la limnologie - Besançon, Franche-Comté, 16-19' Novembre 1992. 


\section{Introduction}

Le seston peut être défini comme l'ensemble des particules en suspension lacustres appartenant au plancton et au trypton. Le trypton rassemble les particules organiques détritiques et minérales (Dussart 1966) ainsi que des complexes néoformés. Les floraisons phytoplanctoniques, les productions autochtones de calcite, les apports terrigènes par les affluents, la décomposition automnale des ceintures végétales et les remises en suspension des sédiments constituent successivement des pics dans les flux de matières en suspension lacustres (Gandais 1989) et participent à la constitution du seston. Dans ce champ très vaste d'investigations, diverses voies de recherche ont été ouvertes concernant l'autochtonie-allochtonie des particules (Zumstein \& Buffle 1989), les mécanismes de précipitation calcique (Kelts \& Hsu 1978, Stabel 1986, KuchlerKrischun \& Kleiner 1990) et les transferts verticaux de matière (Bloesch 1974, Stroffek 1990). D'autres travaux concernent l'analyse microparticulaire des échantillons (Lund et al. 1958, Blanc 1968, Gordon 1970, Landmann 1988).

Dans la recherche de protocoles d'observation et de dénombrement de particules, la difficulté de séparer les fractions vivante et détritique conduit souvent à l'abandon de la frontière entre vivant ou non, pour considérer les particules sous l'angle planctonique ou non. Wirth \& Seifert (1988) identifient pour la mer du Nord, deux types de particules :

- «Détrital-minerogenic Suspended Matter », matrice organo-détritique et minérale essentiellement présente dans les échantillons de fond.

- «Biogenic Suspended Matter », organismes planctoniques et frustules dans divers degré de destruction.

Cette classification simple a l'avantage de fournir une approximation rapide des proportions relatives entre matériel planctonique vivant ou détritique et le reste du matériel en suspension. Il demeure toutefois un problème d'identification et de définition concernant ce « reste » qui représente plus de $50 \%$ de la matière en suspension lacustre (Bloesch et al. 1977, Gandais 1989) et pour lequel on rencontre les termes « détritus » (Bloesch, op. cit.), « terrigène " (Gandais, op. cit.), " matrice organominérale détritique " (Wirth \& Seifert, op. cit.), « fraction non-algale » (Sugiura 1981).
En l'absence de données ántérieures, nous présentons pour 9 lacs du Jura français, les premiers résultats relatifs aux matières en suspension : concentration, composition majeure (perte au feu, calcium), nature (phytoplancton, zooplancton, trypton). Nous abordons l'étude des particules organiques dont la nature et l'abondance sont discutées.

\section{Matériel et méthodes}

\subsection{Les lacs}

Situés au sud-est de la Franche-Comté, ils se répartissent par altitude de 400 à $500 \mathrm{~m}$ pour les lacs de la Combe d'Ain jusqu'à $1000-2000 \mathrm{~m}$ pour les lacs de la haute chaîne (Fig. 1). Les éléments de la paléogéographie régionale, indiquent que les surfaces lacustres se sont considérablement amoindries depuis l'époque post-würmienne (Campy 1982). Sans présumer des phases successives de comblements, l'examen des sédiments récents (Verneaux et al. 1987) met en évidence des pôles sédimentaires carbonatés (Clairvaux), organiques (Les Rousses) ou mixtes (Ilay). La présente analyse quantitative et comparative des matières en suspension, concerne les lacs de profondeur maximale proche ou supérieure à 20 mètres.

\subsection{L'échantillonnage}

Les colonnes d'eau profondes de 9 lacs ont été échantillonnées par pompage entre le 20 août et le 13 septembre 1985 . Le pompage offre une reproductibilité satisfaisante des mesures (Golterman et al. 1983), et le protocole comparatif élaboré, se situe dans la période de désoxygénation critique des systèmes. Afin de limiter l'impact de la variabilité spatiale de la répartition verticale du seston, le prélèvement intègre la hauteur d'eau, au cours d'une remontée de la crépine, à vitesse constante $(5 \mathrm{~m} / \mathrm{mn})$. L'échantillon est constitué de deux prélèvements de 20 à 30 litres d'eau par lac : l'un destiné à la détermination de la concentration en M.E.S., des pourcentages de perte au feu et de calcium particulaire, l'autre à l'analyse microscopique des constituants sestoniques.

\subsection{Les analyses physico-chimiques}

Les mesures de l'oxygène dissous et du potentiel d'oxydo-réduction sont effectuées " in situ " tous les 2 mètres à l'aide d'électrodes sélectives. Pour l'oxygène, un contrôle par la méthode de Winckler est effectué pour les teneurs inférieures à $3 \mathrm{mg} / \mathrm{l}$. 


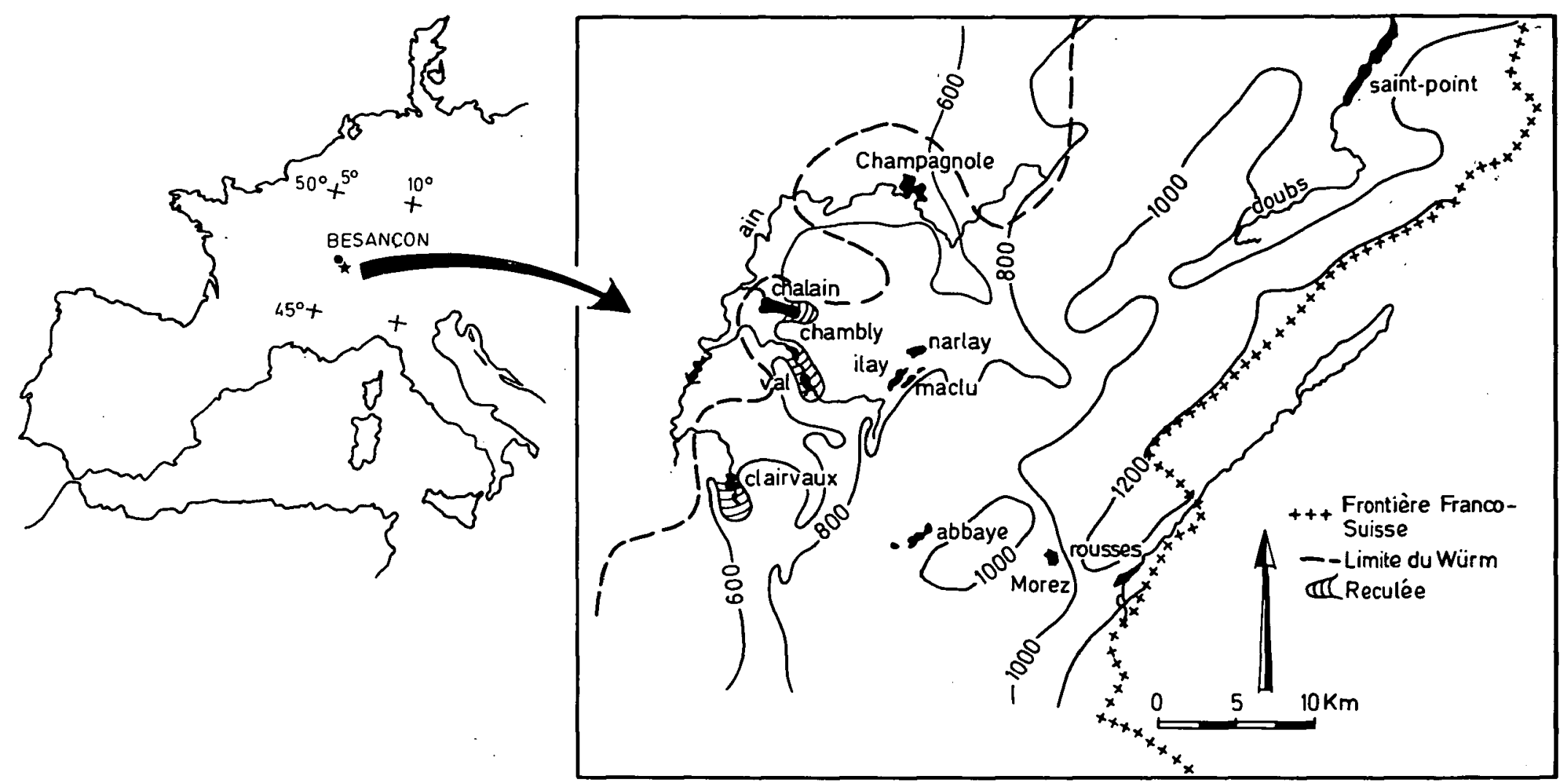

Fig. 1. Localisation des lacs dans la région étudiée.

Fig. 1. Location of lakes in the area studied.

La quantité de matière en suspension est déterminée par gravimétrie (précision $=0,1 \mathrm{mg}$ ), après dépôt sur des filtres Millipore ${ }^{\circledR}$ de $0,45 \mu \mathrm{m}$ de diamètre de pore, et séchage en étuve ventilée à $105^{\circ} \mathrm{C}$. Le pourcentage de matière organique correspond à la perte au feu. Les filtres sont placés à $525^{\circ} \mathrm{C}$ pendant 2 heures (perte moyenne du filtre $=0,055 \%$ du poids initial). Après dissolution du résidu de calcination dans l'acide chlorhydrique $\mathrm{d}=1.16$, le calcium a été dosé au spectromètre par émission de flamme.

\subsection{Les dénombrements particulaires}

Les échantillons sont examinés non fixés au laboratoire, le plus rapidement possible après le prélèvement pour l'identification et le dénombrement des groupes les plus fragiles tels que les Chrysophycées ou les Flagellés. L'échantillon est ensuite filtré sur une colonne de tamis en nylon Blutex d'ouvertures de maille décroissantes $(1600 \mu, 800 \mu, 500 \mu, 355$ $\mu, 237 \mu, 172 \mu, 115 \mu, 40 \mu, 25 \mu$ ). Après un rinçage soigneux, le refus de chaque tamis est conservé au formol neutralisé (Bourrelly 1972). Jusqu'au tamis de $800 \mu$, les observations et les comptages sont effectués au microscope stéréoscopique. En deçà de cette limite de taille, le microscope à inversion offre la solution la mieux adaptée ; l'échantillon est observé dans une cellule de comptage dont le réseau de quadrillage est disposé de manière à ce que le champ de vision du microscope englobe deux lignes successives. Pour chacune des catégories de particules dénombrées, le rapport moyenne/variance a été calculé et sa déviation par rapport à l'unité contrôlé par la méthode du « Chi deux ". Les changements de variables appropriées ont été effectuées pour que les données soient conformes à une distribution régulière (Elliott \& Décamps 1973).

\section{Résultats}

\subsection{Physico-chimie des eaux et des suspensions}

La figure 2 présente les lacs étudiés, en fonction de leur altitude et du degré d'anaérobiose de leurs eaux évalué par un nouvel indice de désoxygénation $\left(e_{r} 20\right)$ qui exprime la hauteur d'eau relative où le pourcentage de saturation en oxygène est inférieur à $20 \%$ (seuil de libération du phosphore bloqué dans les complexes sédimentaires). Sur les 9 lacs échantillonnés, seuls les lacs Maclu et Saint-Point bénéficient d'une saturation en oxygène supérieure à $20 \%$ sur toute la tranche d'eau. 
4 lacs (Rousses, Abbaye, Ilay, Narlay) sont en situation de lacs froids et désoxygénés, et 3 lacs en posi- tion de lacs chauds en anaérobiose modérée (Chalain, Clairvaux, Val).

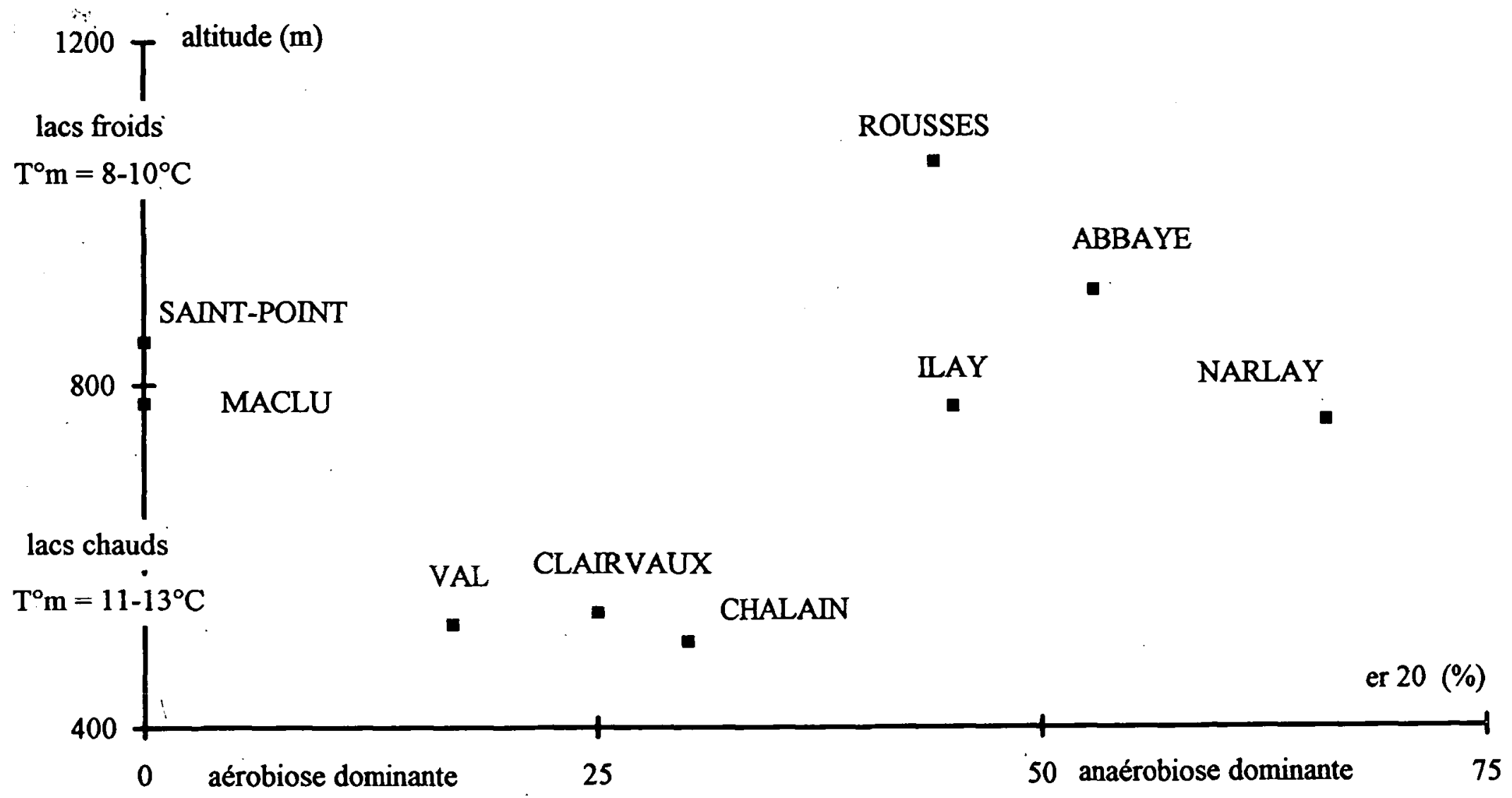

Fig. 2. Positionnement des lacs étudiés en fonction de l'altitude $(\mathrm{m})$ et du degré de désoxygénation de l'hypolimnion. $\mathrm{e}_{\mathrm{r}} 20$ (\%) : hauteur d'eau relative (en $\%$ ) où le pourcentage estival de saturation en oxygène est inférieur à $20 \%$.

Fig .2. Location of lakes as a function of altitude $(\mathrm{m})$ and oxygen depletion of hypolimnion. $\mathrm{e}_{\mathrm{r}} 20(\%)$ : relative water depth where $\%$ sat. $\mathrm{O}_{2}<20 \%$.

Les concentrations en matières en suspension à la fin de l'été se situent dans une gamme de valeurs allant de $0,89 \mathrm{mg} / 1$ à $5,51 \mathrm{mg} / 1$ (Tableau 1) qui se rapprochent de celles rassemblées par Sturm (1985) pour les lacs alpins ou péri-alpins à production autochtone dominante (calcite + phytoplancton). Les variations du pourcentage de $\mathrm{CaCO}_{3}$ autour de la moyenne de 14,62 \% sont sensibles et s'échelonnent de 4 à $8 \%$ pour l'Abbaye, Maclu, Les Rousses et Narlay, de 10 à $20 \%$ pour le Val, Ilay et Châlain et de 30 à $35 \%$ pour les lacs de St-Point et de Clairvaux. Les concentrations en matière organique particulaire évaluées par la perte au feu sont comprises entre $0,36 \mathrm{mg} / \mathrm{l}$ et $2,65 \mathrm{mg} / 1$, et séparent d'un facteur 10 les extrêmes que sont les lacs de Narlay et du Maclu. En terme de pourcentage, les écarts réduits ( $33 \%$ à $48 \%$ ) indiquent que la perte au feu constitue dans les 9 échantillons analysés une frac- tion relativement constante du poids de matières en suspension totales. La figure 3 laisse apparaître deux groupes de lacs, distingués par leurs concentrations. en MES, et dont Ilay serait un représentant mixte.

\subsection{Nature des particules}

Parmi les suspensoïdes lacustres, les formes les plus abondamment rencontrées dans les lacs sont présentées au tableau 2 . On sépare le plancton vivant (groupes 1 et 2) de la fraction détritique. Les particules organiques appartiennent au trypton et sont constituées, soit de fragments organiques d'origine diverse : allochtones stricts (provenant d'organismes terrestres), planctoniques ou littoraux (ceintures végétales), soit d'associations composites organominérales de teinte et de forme diverses (groupes 3 et 4). 
Tableau 1. Concentrations en matières sèches en suspension (MES, $\mathrm{mg} / \mathrm{l})$, matières organiques particulaires (PF, $\mathrm{mg} / \mathrm{l}$ et $\% \mathrm{~m} . \mathrm{s})$, calcium particulaire $\left(\mathrm{Ca}, \% \mathrm{~m} . \mathrm{s}\right.$.), carbonate de calcium $\left(\mathrm{CaCO}_{3}=2,5 * \mathrm{Ca}, \% \mathrm{~m} . \mathrm{s}\right.$.) pour le seston de 9 lacs jurassiens échantillonnés entre le 22 août et le 11 septembre 1985.

Table 1. Dry particulate suspended matter (MES, mg/l), organic particulate matter (Ignition Loss, mg/l and \% d.m.), particulate calcium ( $\mathrm{Ca}, \%$ d.m.), calcium carbonate $\left(\mathrm{CaCO}_{3}=2,5 * \mathrm{Ca}, \%\right.$ d.m.). Seston from 9 lakes of the French Jura sampled between August 20 th 1985 and September 11 th 1985.

\begin{tabular}{|c|c|c|c|c|c|c|c|c|}
\hline dates & codes & lacs & $\begin{array}{c}\mathrm{z} \\
\mathrm{m}\end{array}$ & $\begin{array}{l}\mathrm{MES} \\
\mathrm{mg} / 1\end{array}$ & $\begin{array}{c}\mathrm{PF} \\
\mathrm{mg} / \mathrm{l}\end{array}$ & $\begin{array}{c}P F \\
\%\end{array}$ & $\begin{array}{c}\mathrm{Ca} \\
\%\end{array}$ & $\begin{array}{c}\mathrm{CaCO}_{3} \\
\%\end{array}$ \\
\hline $11 / 09 / 85$ & NAR & Narlay & 39 & 5,51 & 2,65 & 48,13 & 3,24 & 8,10 \\
\hline $22 / 08 / 85$ & ABB & Abbaye & 18 & 4,66 & 1,98 & 42,49 & 1,88 & 4,70 \\
\hline $07 / 09 / 85$ & ROU & Rousses & 18 & 3,90 & 1,47 & 37,68 & 2,38 & 5,95 \\
\hline $05 / 09 / 85$ & VAL & Val & 24 & 3,64 & 1,37 & 37.56 & 4,40 & 11,00 \\
\hline $28 / 08 / 85$ & CLA & Clairvaux & 20 & 2,72 & 0,91 & 33,33 & 13,72 & 34,30 \\
\hline $23 / 08 / 85$ & ILA & Ilay & 28 & 2,02 & 0,80 & 39,54 & 5,24 & 13,10 \\
\hline $20 / 08 / 85$ & SPO & St-Point & 33 & 1,90 & 0,63 & 33,00 & 12,37 & 30,90 \\
\hline $02 / 09 / 85$ & $\mathrm{CHA}$ & Châlain & 30 & 1,53 & 0,61 & 39,66 & 7,19 & 17,98 \\
\hline \multirow[t]{4}{*}{$30 / 08 / 85$} & MAC & Maclu & 25 & 0,89 & 0,36 & 40,40 & 2,22 & 5,55 \\
\hline & & \multicolumn{2}{|c|}{ moyenne } & 2,97 & 1,20 & 39,10 & 5,85 & 14,62 \\
\hline & & \multicolumn{2}{|c|}{ écart-type } & 1,46 & 0,70 & 4,35 & 4,17 & 5,00 \\
\hline & & \multicolumn{2}{|c|}{ variance } & 2,13 & 0,49 & 18,90 & 17,39 & 25,00 \\
\hline
\end{tabular}

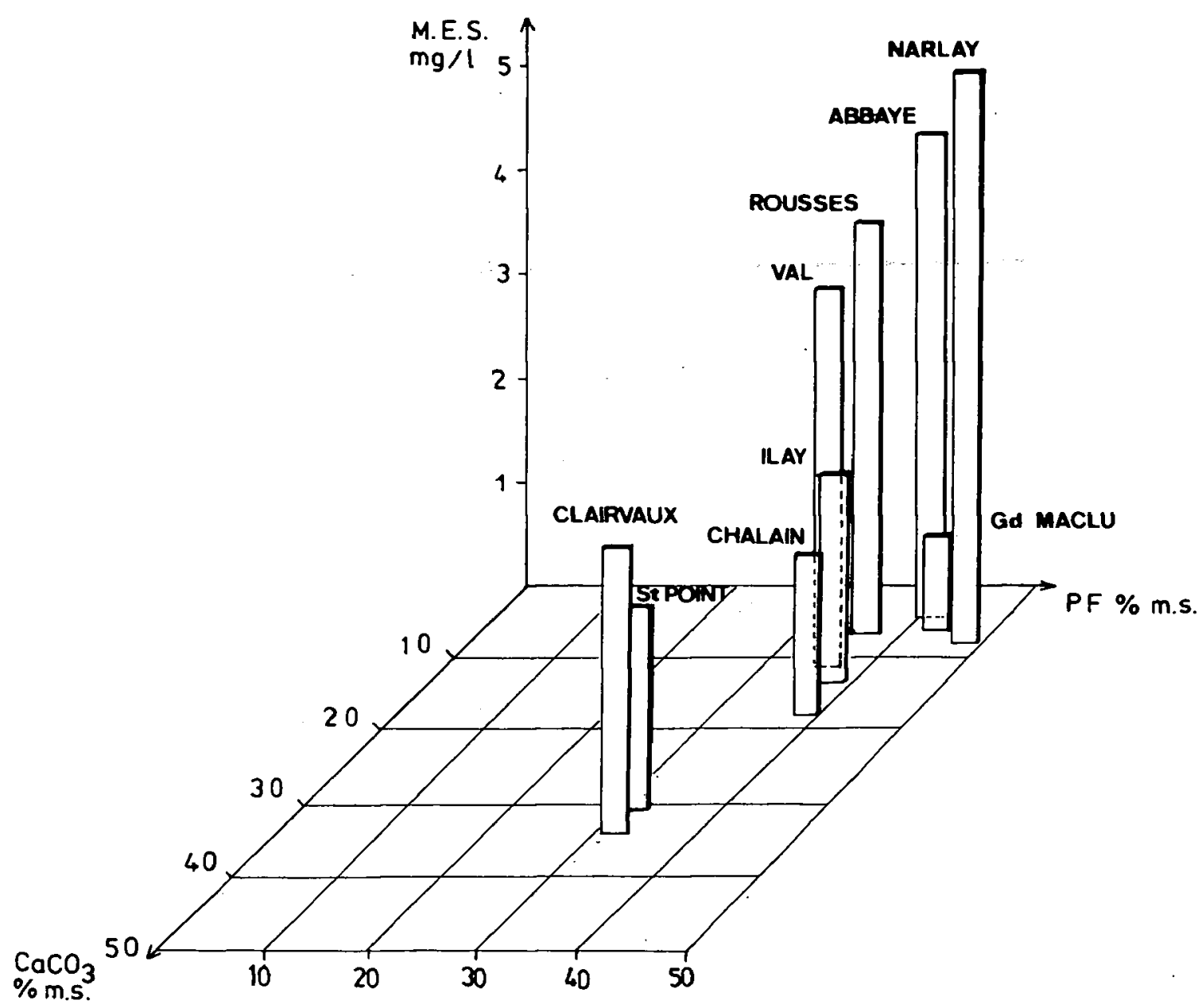

Fig. 3. Distribution des échantillons de MES de 9 lacs jurassiens en fonction de la concentration en matière sèche, du pourcentage de $\mathrm{PF}$ et du pourcentage de $\mathrm{CaCO}_{3}$.

Fig. 3. Distribution of Suspended Matter samples from 9 Lakes of the French Jura depending on concentration of dry matter, Ignition Loss (\% d.m.) and $\mathrm{CaCO}_{3}(\%$ d.m.). 
Tableau 2. Classification des suspensoïdes lacustres retenus pour l'analyse microparticulaire du seston de neuf lacs du Jura français. Table 2. Classification of Lacustrine Suspended Particle matter of nine lakes of the French Jura.

\begin{tabular}{|lll|}
\hline PLANCTON & GROUPE 1: & le phytoplancton vivant \\
& GROUPE 2: & le zooplancton vivant. \\
GRYPTON & GROUPE 3 : & $\begin{array}{l}\text { les éléments figurés qui incluent } \\
\text { les fragments organo-détritiques, les. } \\
\text { particules d'origine anthropique, et les } \\
\text { particules minérales. }\end{array}$ \\
GROUP 4: & $\begin{array}{l}\text { les associations composites plus ou } \\
\text { moins condensées de matière organique, } \\
\text { de colloïdes argileux et de nannocristaux } \\
\text { de calcite. }\end{array}$ \\
\hline
\end{tabular}

Le groupe 3 rassemble les " éléments figurés 》 définis comme les particules identifiables n'ayant pas fait l'objet de remaniements. Nous avons inclus dans ce groupe les fragments organo-détritiques qui constituent, selon la terminologie de Gordon (1970), un synonyme des détritus de Wetzel (1983), c'est-à-dire des particules formées par la fragmentation de tissus organiques (clastes). Nous avons séparé les clastes d'origine autochtone : phytoclastes et zooclastes (Fig. 4 a, b), les macrophytoclastes (Fig. 4 c, d, e), et les fragments d'origine strictement allochtone (Fig. $4 \mathrm{f}, \mathrm{g}, \mathrm{h}$ ). Nous avons également rencontré, dans la plupart des échantillons, des fibres cellulosiques colorées d'origine anthropique qui nous ont incité à créer une catégorie « fragments allochtones anthropiques ». Manheim et al. (1972) décrivent des fibres similaires au large du golfe de Mexico. Rentreraient également dans cette catégorie des particules sphériques d'origine industrielle rencontrées en mer du Nord au large de l'embouchure du Rhin (Hölemann \& Wirth 1988). Les phytoclastes et les particules minérales sont particulièrement fins (70 à $80 \%$ du nombre compris entre 25 et $40 \mu \mathrm{m}$ ). Les zooclastes et les macrophytoclastes sont également bien représentés dans la fraction la plus fine ( $>$ $60 \%$ ), mais sont également présents dans les fractions les plus grossières (macrophytoclastes : 0,1 $\%>1600 \mu \mathrm{m}$, zooclastes : $0,1 \%>500 \mu \mathrm{m})$.

Le groupe 4 rassemble les associations organominérales composites. Sur les quatre catégories identifiées, les deux principales, les agrégats et les flocons ont déjà fait l'objet de descriptions. Riley (1963) puis Gordon (op. cit.) décrivent les agrégats (Fig. $5 \mathrm{c}, \mathrm{d}$ ) comme des associations de phases organique et minérale en proportions variables, à contours peu définis. Cette définition recoupe celle donnée par Hölemann \& Wirth (1988) pour les microflocs de la mer du Nord. Leur couleur s'apparente à l'ocre-brun. Les flocons (Fig. 5 e, f) sont définis par opposition aux agrégats comme étant de couleur brun-noir, au contour net, circulaire ou allongé. Ils pourraient constituer la phase non algale des échantillons de Sugiura (1981). 57 \% des flocons sont compris entre 40 et $115 \mu \mathrm{m}$, tandis que $54 \%$ des agrégats sont situés entre 25,5 et $40 \mu \mathrm{m}$. Les amoncellements d'éléments figurés (Fig. $5 \mathrm{a}, \mathrm{b}$ ) sont des enchevêtrements d'éléments figurés en voie de décomposition avec, la plupart du temps, présence de bactéries. Les macroflocs composites (Fig. $5 \mathrm{~g}$, h) sont formés par des enchevêtrements des trois catégories décrites précédemment. Le terme agrégats, couramment utilisé (Otsuki \& Hanya 1972, Paerl 
a
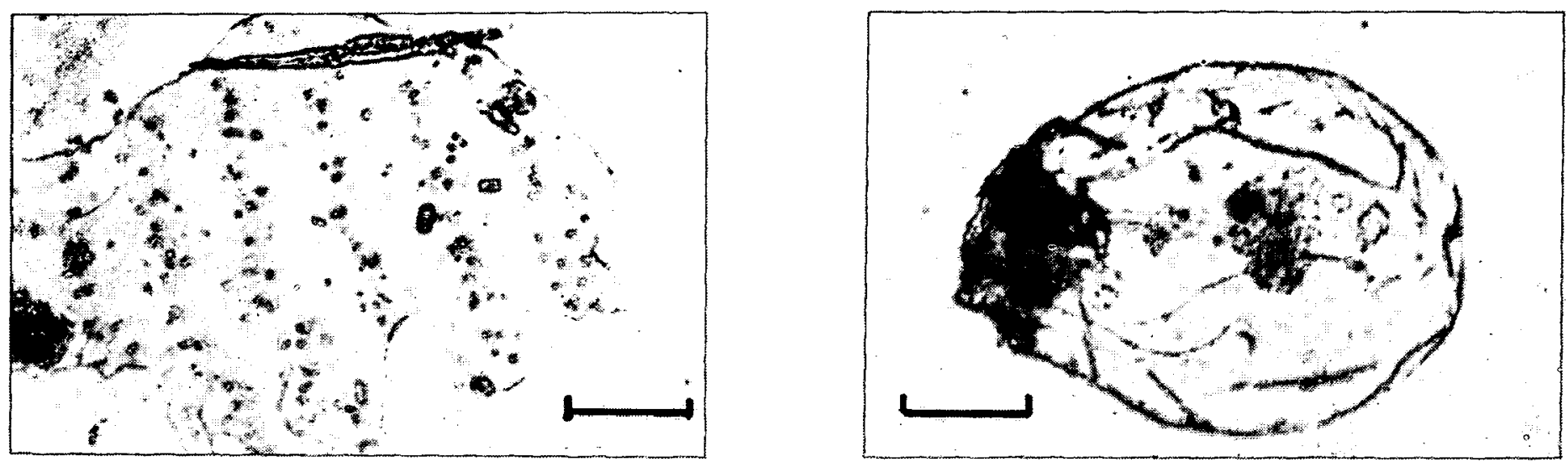

b

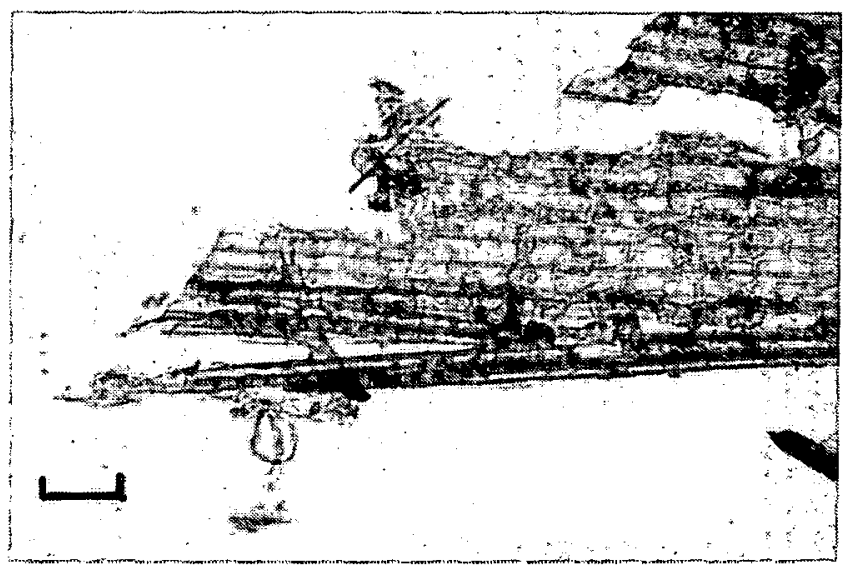

d

C
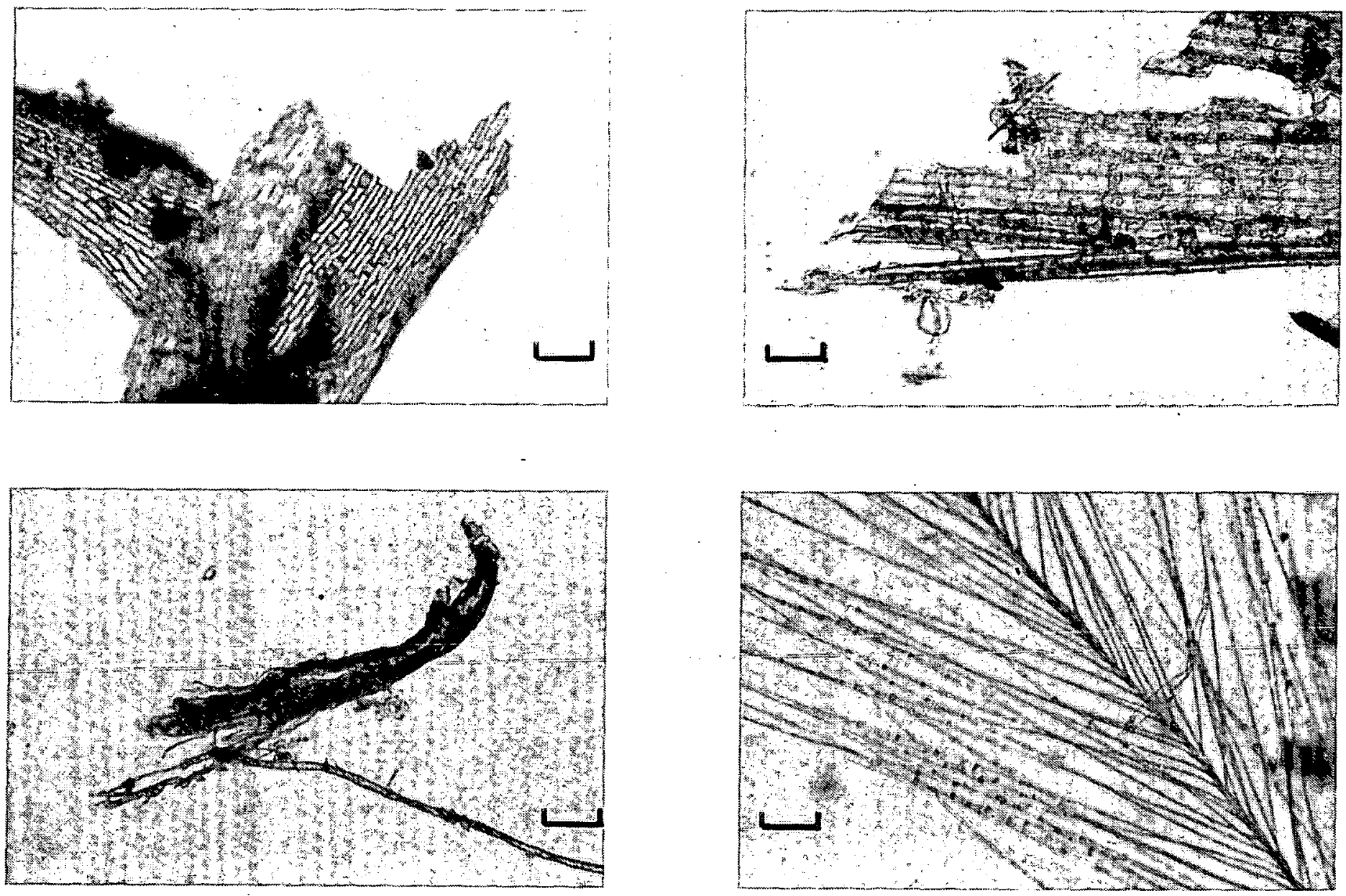

e
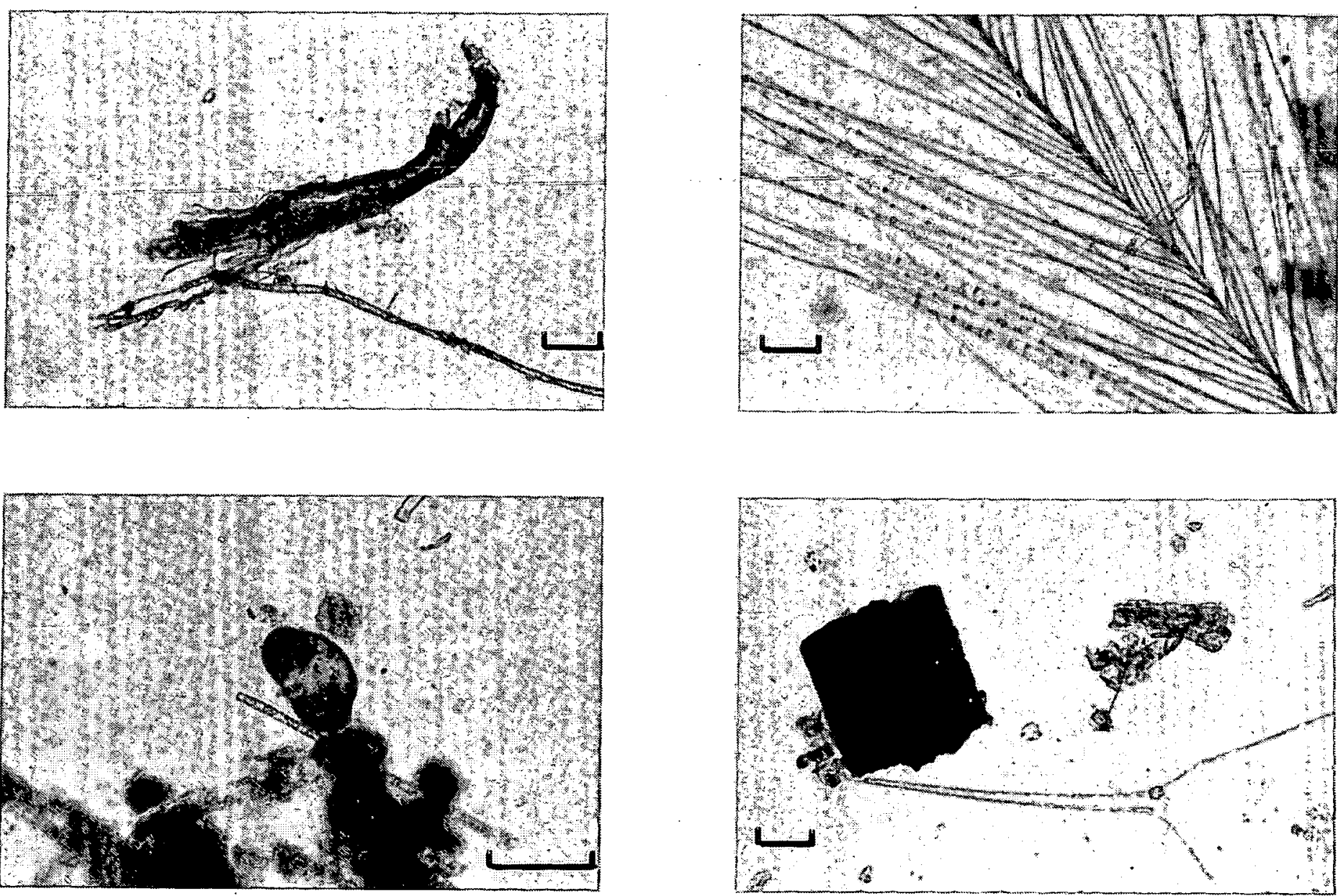

h

Fig. 4. Eléments figurés : a) fragment de Cladocère, b) fragment de Rotifère, c) d) e) macrophytoclastes, f) inflorescence, g) pollen, h) fragment ligneux. [ $]=100 j \mathrm{~m}$.

Fig. 4. Detritus : a) Cladocera fragment, b) rotifer chip, c) d) e) macrophyte chip, f) bloom chip, g) pollen chip, h) wood chip. [ _ _ ] $=100^{\circ} \mu \mathrm{m}$. 
a

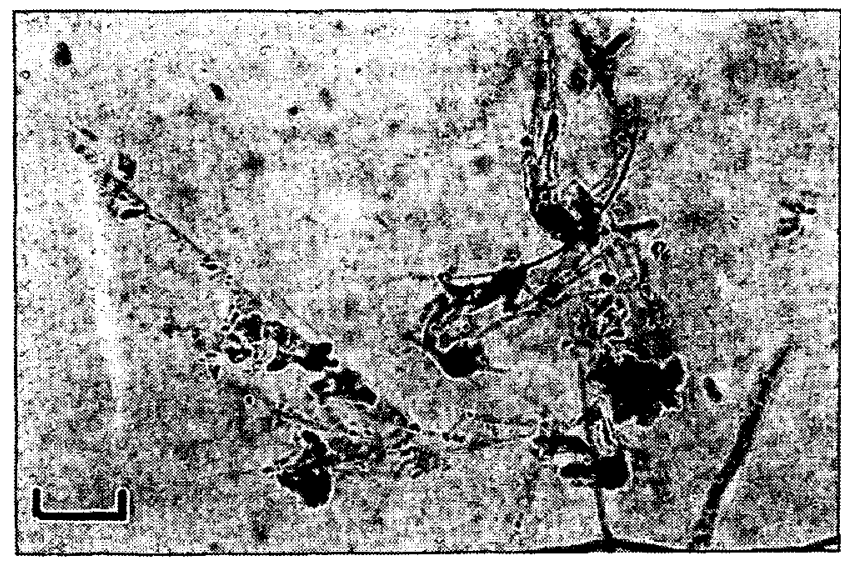

c

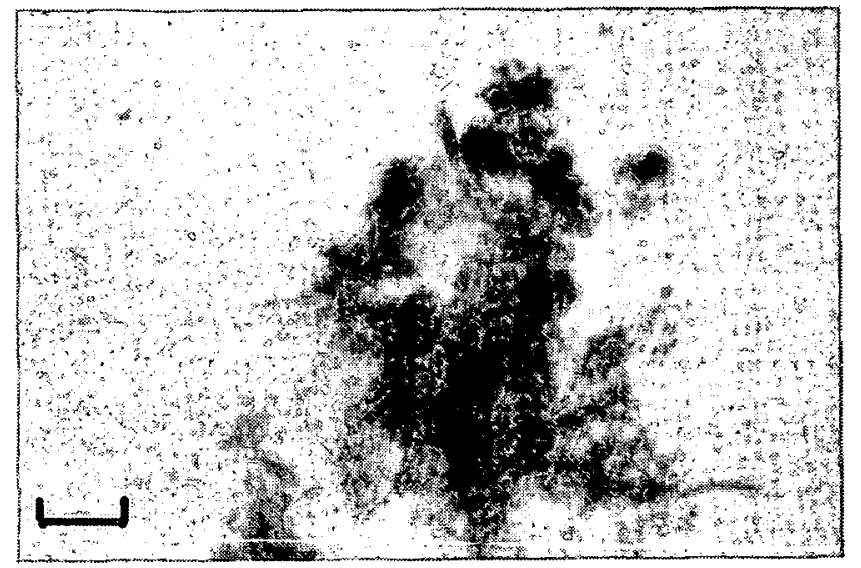

e
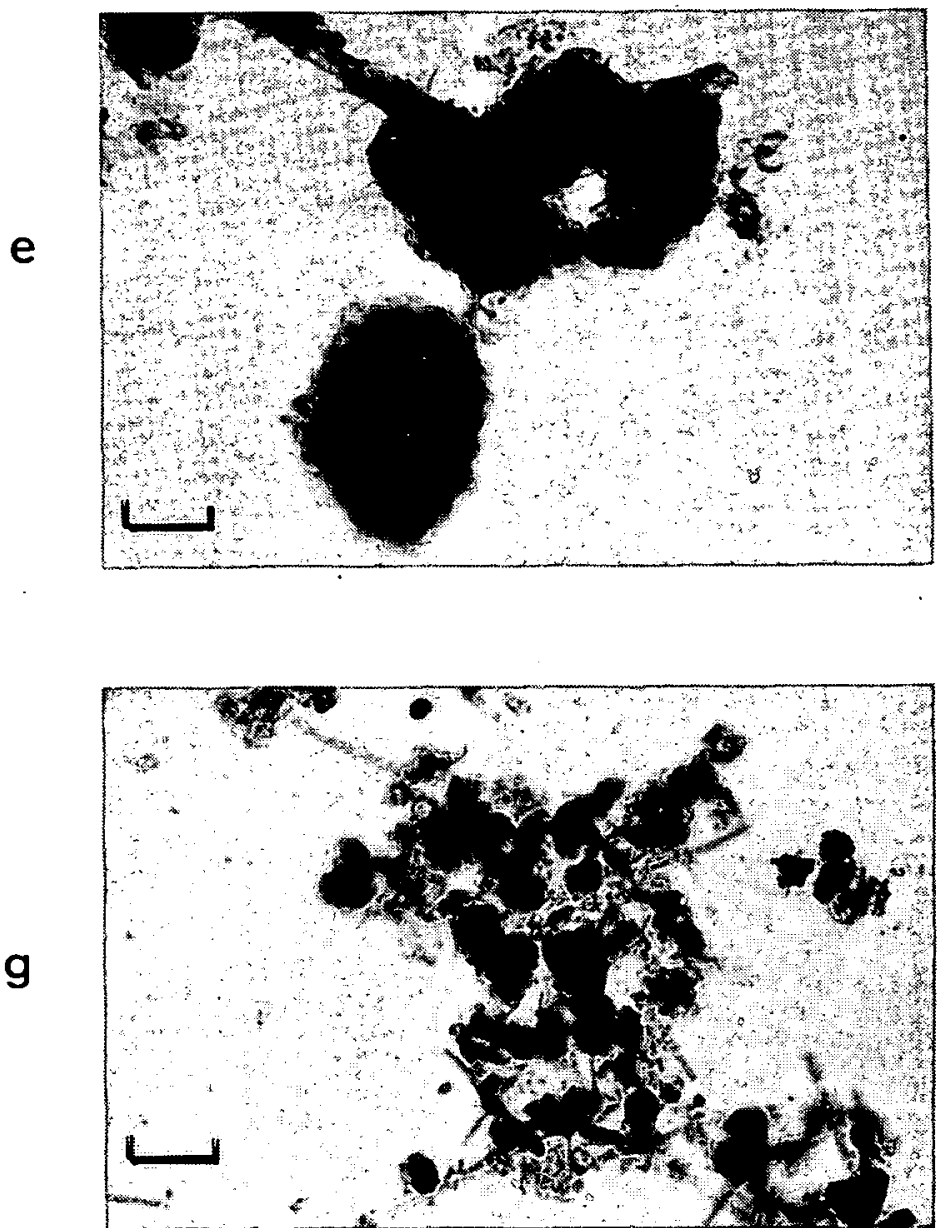

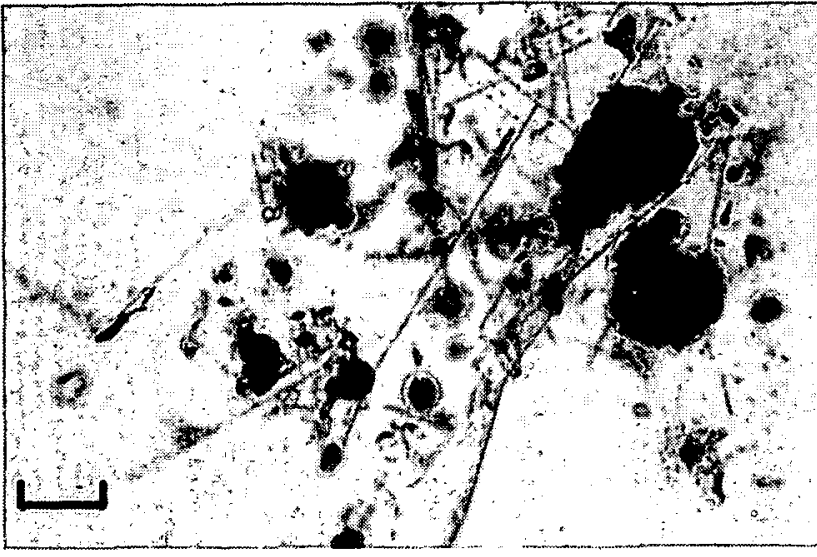

b

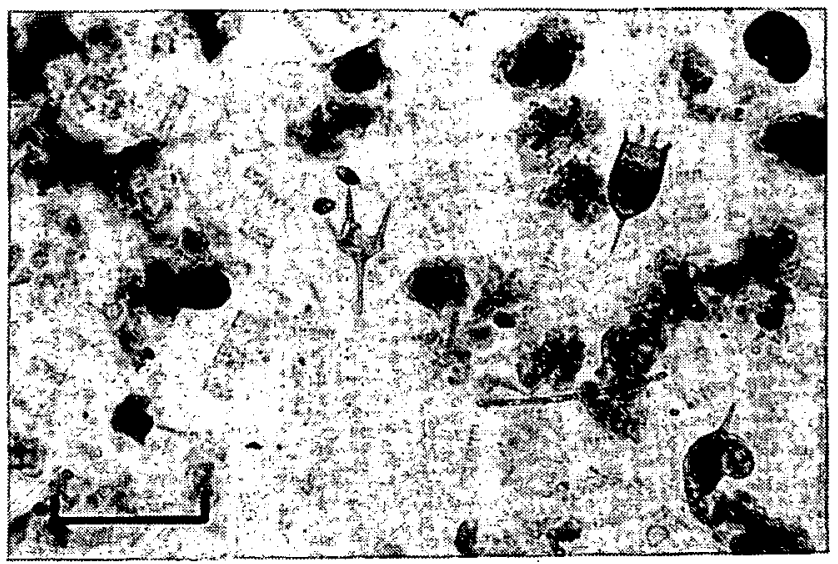

d
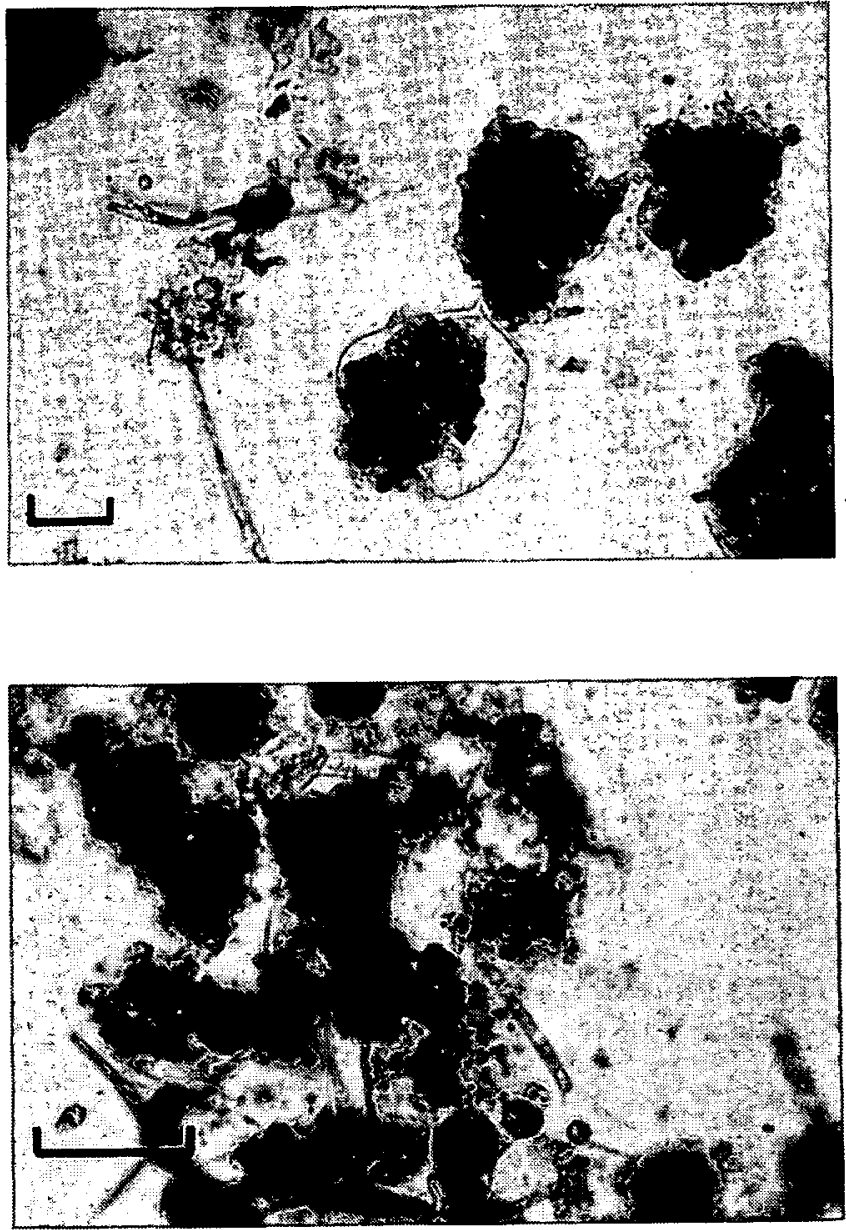

Fig. 5. Associations organo-minérales composites : a) b) amoncellement d'éléments figurés, c) d) agrégats, e) f) flocons, g) h) macroflocs composites. [ ] $=100 \mu \mathrm{m}$.

Fig. 5. Heterogeneous mineral and organic compounds : a) b) agglomerate detritus, c) d) aggregates, e) f) flakes, g) h) heterogeneous big flakes. [ ] $=100 \mu \mathrm{m}$. 
1974, Hunt 1982), peut être rapporté au terme associations organo-minérales composites, utilisé pour définir le groupe 4, l'absence de description ou d'illustration ne permettant pas plus de précision.

\subsection{Analyse des abondances}

Les dénombrements particulaires indiquent que deux lacs sont le siège de proliférations algales : Oscillatoria rubescens De Candolle, au lac de Narlay et Sphaerocystis sp. au lac du Val. L'abondance du phytoplancton dans les autres lacs est similaire et proche de $0,5.10^{4}$ cellules/l. Pour l'ensemble des lacs, le phytoplancton est dominant, de 50 à $80 \%$, sauf dans le cas du lac d'Illay où il ne représente que $43,3 \%$. Le zooplancton est toujours largement minoritaire, de l'ordre de $1,5 \%$ en moyenne. Le trypton représente $30 \%$ en moyenne du nombre de particules en suspension (Tablèau 3, Fig. 6 A).

Sur la moyenne des échantillons, les zooclastes $(38,3 \%)$ se partagent avec les macrophytoclastes $(40,8 \%)$ la dominante numérique des éléments figurés organiques en suspension. L'analyse des abondances (Fig. 6 B) montre que les zooclastes sont particulièrement nombreux dans les échantillons du Val (1767 particules/litre), lac caractérisé par la prolifération d'une Chlorophycée de petite taille (8-16 $\mu \mathrm{m})$ Sphaerocystis $s p$. et entretenant un zooplancton abondant à base de Protozoaires, Rotifères et Copépodes. Les macrophytoclastes sont les plus nombreux dans les échantillons du lac d'Illay (1008 part/l) où ils dominent à $80 \%$ le nombre des élé- ments figurés organiques. Les phytoclastes constituent la plus faible fraction des éléments figurés organiques, environ $10 \%$, exception faite des lacs de l'Abbaye et de Saint-Point dans lesquels se distinguent les clastes de Diatomées (Asterionella formosa Hassal, Fragilaria crotonensis Kitton). Les plus faibles valeurs d'abondance et de pourcentage en éléments figurés sont rencontrées au lac de Narlay, siège d'une fleur d'eau à Oscillatoria rubescens très pauvre en zooplancton, et Clairvaux et Chalain, lacs où les algues Chrysophytes et Pyrrhophytes, dominantes, accompagnent un zooplancton de petite taille (Protozoaires, Rotifères).

Parmi les associations composites organominérales (Fig. $6 \mathrm{C}$ ), les agrégats et les flocons sont les particules les plus fréquemment observées dans tous les échantillons. Les accumulations d'éléments figurés et les macroflocs composites, considérés comme catégories annexes, ne sont dénombrés que dans quelques lacs et en abondance restreinte. Les agrégats constituent la grosse majorité des associations organo-minérales (71,3\% en moyenne) avec des abondances variant de 150 part/l à Saint-Point à $3221 \mathrm{part} / 1$ au lac d'Illay. Le nombre des flocons concorde avec les teneurs en MES et PF les plus élevées des échantillons analysés, laissant supposer une densité assez forte et une nature très organique de cette famille de particules. Les abondances maximales sont relevées dans les lacs à forte production planctonique (Narlay, Le Val), et dans les lacs de tourbière (Les Rousses, l'Abbaye).

Tableau 3. Groupes des constituants du seston, par lac et en pourcentage du nombre total de particules dénombrées.

Table 3. Percentile of each group of Lacustrine Suspended Particles (percentile of total number of particles).

NAR ABB ROU VAL CLA ILA SPO CHA MAC $\quad \begin{gathered}\text { moyenne } \\ \text { générale }\end{gathered}$

GROUPE $1(\%)$

phytoplancon

GROUPE 2 (\%)

zooplancton

GROUPE $3(\%)$

éléments figurés

GROUPE $4(\%)$

associations organo-

minérales composites

$\begin{array}{rrrrrrrrrr}78,1 & 58,8 & 49,3 & 82,8 & 68,4 & 43,3 & 55,8 & 78,5 & 50,3 & 67,2 \\ 0,1 & 0,2 & 1,7 & 0,9 & 5,8 & 1,3 & 0,8 & 2,3 & 1,2 & 1,2 \\ 4,4 & 18,3 & 8,4 & 11 & 7 & 19,3 & 26,8 & 5,1 & 17,1 & 11,7 \\ 17,4 & 22,7 & 40,7 & 5,3 & 18,7 & 36,1 & 16,7 & 14 & 31,4 & 19,9\end{array}$



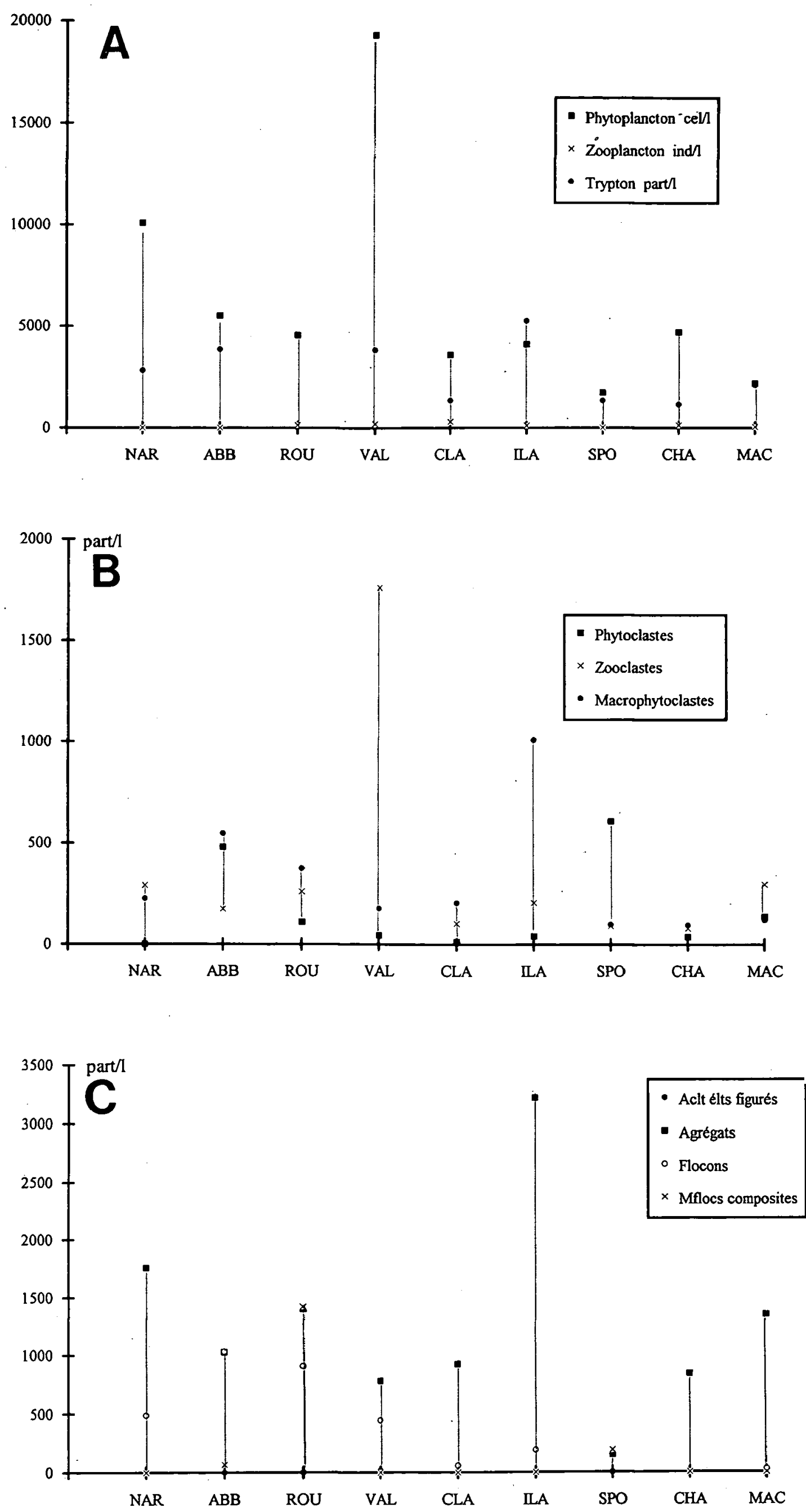


\subsection{Relations}

\subsubsection{Corrélations interparticulaires}

L'examen des corrélations interparticulaires est effectué dans le but de valider les catégories présentées au tableau 2, sans que l'information relative aux causalités des relations observées ne soit recherchée. L'analyse effectuée, tous échantillons confondus, permet de positionner les particules organiques corrélativement aux autres catégories particulaires (zooplancton, phytoplancton) dont nous apprécions mieux la signification écologique globale. La figure 7, élaborée à partir de la matrice des corrélations (Tableau 4) représente schématiquement les corrélations positives les plus fortes observées pour chacune des variables. Considérer les groupes d'algues comme points de départ des relations constitue, selon toute vraissemblance, la seule manière de satisfaire à l'exigence préalable que les flèches relationnelles ne puissent s'entrecroiser. 4 types d'échantillons sont identifiés :

- Type I : Les Chrysophytes, Pyrrhophytes s'associent positivement au petit zooplancton, (protozoaires $r=+0,60$, rotifères $r=+0,64$ ), et aux amoncellements d'éléments figurés dont la composition hétérogène apparaît dans les corrélations positives avec lés zooclastes $(r=+$ $0,58)$, les particules minérales $(r=+0,43)$ et le phytoplancton total $(\mathrm{r}=+0,54)$.

- Type II : Les Chlorophytes se corrèlent aux fragments totaux $(r=+0,72)$, aux Copépodes $(r=+0,64)$, et aux particules minérales $(\mathrm{r}=+0,72)$, rappelant l'intervention possible de ce groupe d'algues dans la nucléation des cristaux de calcite (Stabel 1986).

- Type III : Les Diatomées sont peu liées au reste du plancton, à l'exception des Cyanophycées ( $\mathrm{r}$ $=+0,42$ ), elles présentent des corrélations négatives fortes avec les Chrysophytes, Pyrrhophytes $(\mathrm{r}=-0,56)$, les Rotifères $(\mathrm{r}=$ $-0,75)$ et le zooplancton total $(r=-0,67)$. Elles sont fortement corrélées aux flocons $(r=$ $+0,86)$.

- Type IV : Les Cyanophycées présentent une variation positive avec les Diatomées, conjointement à une réduction du zooplancton $(\mathrm{r}=-$ $0,45)$. Elles sont principalement corrélées aux associations organo-minérales composites $(\mathrm{r}=$ $+0,72)$, aux macrophytoclastes $(r=+0,52)$ et aux agrégats $(r=+0,63)$.

Fig. 6. Dénombrement particulaire (part/1) du seston de 9 lacs du Jura français. Compositions obtenues entre le 20 août et le 13 septembre 1985 par prélèvement intégré.
A) seston global (phytoplancton, zooplancton, trypton).
B) éléments figurés (phytoclastes, zooclastes, macrophytoclastes).
C) association organo-minérales (amoncellements d'éléments figurés, agrégats, flocons, macroflocs composites).

Fig. 6. Particles count (number/it) from seston of 9 lakes of the French Jura, sampled between August 20th 1985 and September 13 th 1985.

A) total seston (phytoplankton, zooplankton and trypton).

B) detritus (phytoplankton fragment, zooplankton fragment, macrophyte fragment).

c) heterogeneous mineral and organic compounds (agglomerate detritus, aggregats, flakes, heterogeneously big flakes). 


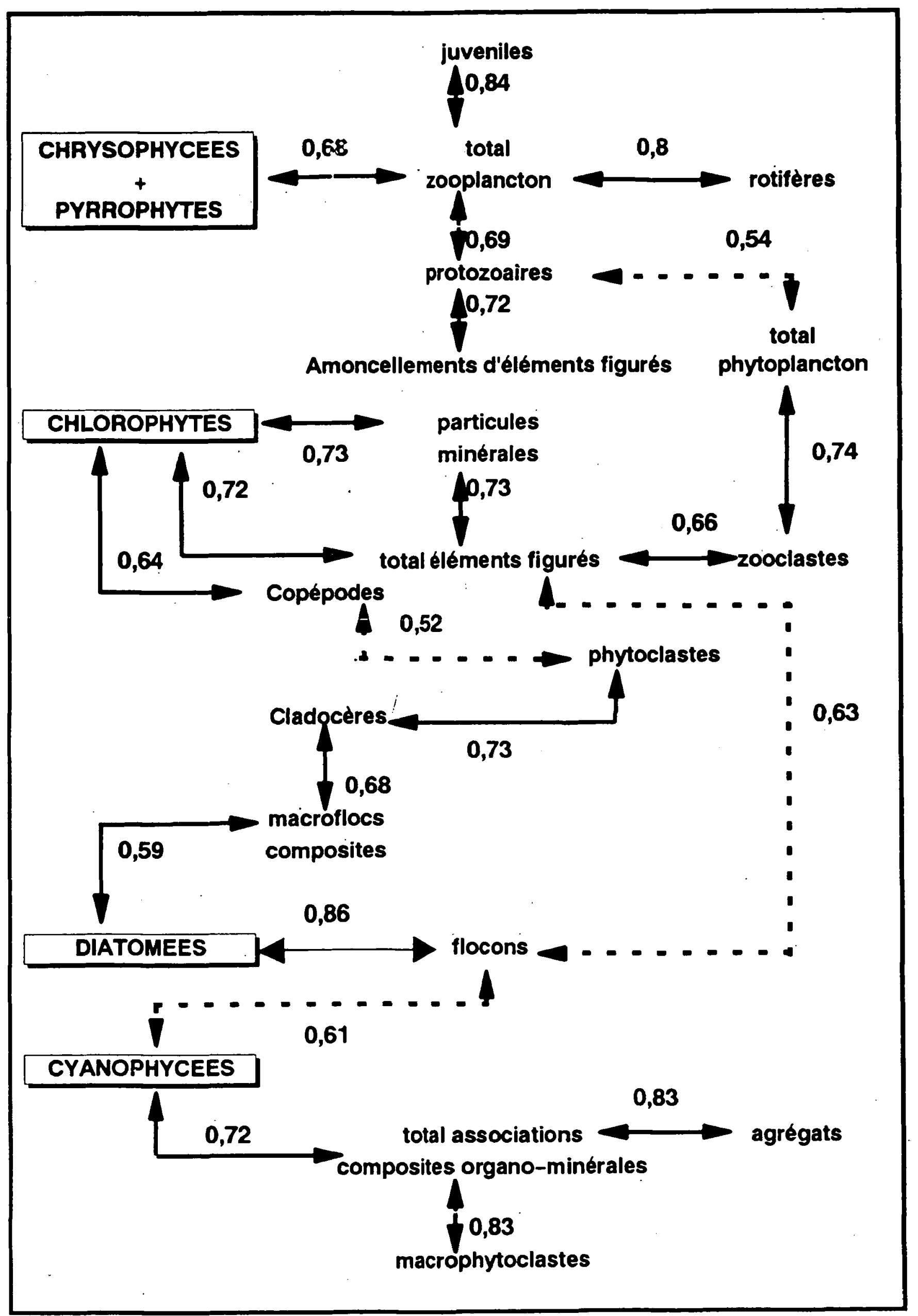

Fig. 7. Représentation schématique des principales corrélations positives entre les constituants de la matière en suspension lacustre. Chaque variable est liée à la suivante par sa valeur de corrélation la plus forte observée dans la matrice (trait plein). Chaque type statistique, identifié par chaque groupe d'algue, est lié au suivant par le couple de variables présentant la plus forte valeur de corrélation (trait pointillé).

Fig. 7. Positive correlation diagram. Each particle is joined to the other with the strongest value of correlation (full line). Statistical groups, represented by algae groups, are joined by the couple of particles with the strongest value of correlation (dotted line). 
Tableau 4. Matrice des corrélations interparticulaires.

Table 4. Correlation matrix.

\begin{tabular}{|c|c|c|c|c|c|c|c|c|c|c|c|c|c|c|c|c|c|c|c|c|c|}
\hline & 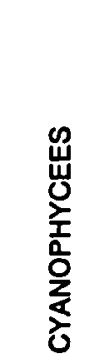 & 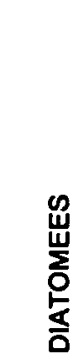 & 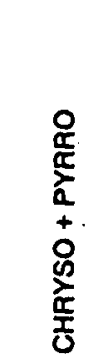 & 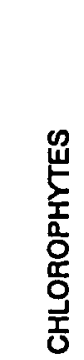 & $\begin{array}{l}z \\
0 \\
0 \\
z \\
5 \\
0 \\
0 \\
0 \\
\frac{1}{0} \\
\frac{1}{2} \\
0 \\
0 \\
0\end{array}$ & 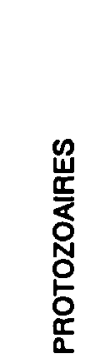 & 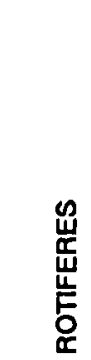 & 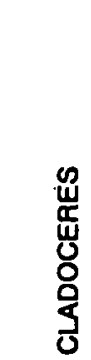 & 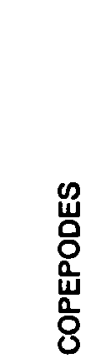 & 总 & 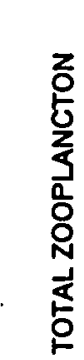 & 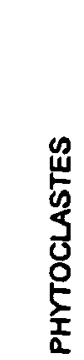 & 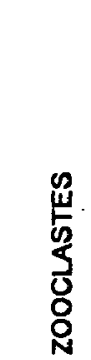 & 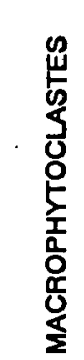 & 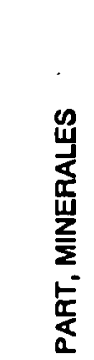 & 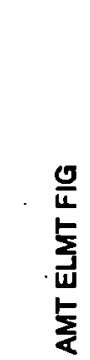 & 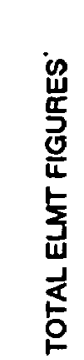 & 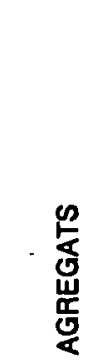 & 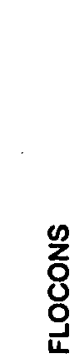 & 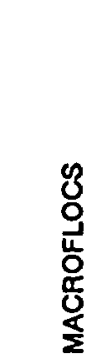 & $\begin{array}{l}z \\
\frac{z}{5} \\
0 \\
\frac{\pi}{0} \\
\frac{1}{0} \\
5\end{array}$ \\
\hline CYANOPHYCEES & 1,00 & 0,42 & $-0,09$ & $-0,09$ & 0,41 & $-0,02$ & $-0,29$ & $-0,17$ & $-0,49$ & $-0,35$ & $-0,45$ & $-0,44$ & 0,57 & 0,52 & 0,26 & 0,04 & 0,43 & $0,63^{\circ}$ & 0,61 & $-0,11$ & 0,72 \\
\hline DIATOMEES & & 1,00 & $-0,56$ & $-0,07$ & 0,30 & $-0,19$ & $-0,75$ & 0,10 & $-0,40$ & $-0,39$ & $-0,67$ & $-0,09$ & 0,22 & 0,32 & $-0,04$ & $-0,21$ & 0,46 & $-0,17$ & 0,86 & 0.59 & 0,29 \\
\hline CHRYSO + PYRRO & & & 1,00 & $-0,06$ & 0,38 & 0,60 & 0,64 & $-0,06$ & $-0,03$ & 0,38 & 0,68 & $-0,25$ & 0.23 & $.0,02$ & 0,01 & 0,34 & -0.25 & 0,42 & 0,28 & -0.29 & 0,20 \\
\hline CHLOROPHYTES & & & & 1,00 & 0,15 & $-0,09$ & 0,11 & 0,22 & 0,64 & 0,51 & 0,30 & 0,17 & 0,48 & 0,10 & 0,73 & 0,36 & 0,72 & $-0,03$ & $-0,03$ & $-0,10$ & $-0,02$ \\
\hline TOTAL PHYTOPLANCTON & & & & & 1,00 & 0,36 & 0,07 & $-0,46$ & $-0,30$ & $-0,17$ & 0,00 & $-0,79$ & 0,74 & 0.19 & 0,36 & 0,54 & 0,38 & 0,32 & 0,40 & $-0,30$ & 0,30 \\
\hline PROTOZOAIRES & & & & & & 1,00 & 0,53 & $-0,21$ & $-0,06$ & 0,59 & 0,69 & $-0,29$ & 0,39 & 0,05 & $-0,15$ & 0,71 & 0,06 & 0,02 & 0,22 & 0,01 & 0,06 \\
\hline ROTIFERES & & & & & & & 1,00 & $-0,08$ & 0,20 & 0,44 & 0,80 & $-0,28$ & 0,11 & $-0,18$ & $-0,12$ & 0,49 & $-0,30$ & 0,25 & $-0,52$ & $-0,50$ & $-0,06$ \\
\hline CLADOCERES & & & & & & & & 1,00 & 0,28 & 0,36 & 0,13 & 0,73 & $-0,30$ & 0,35 & $-0,14$ & $-0,51$ & 0,10 & 0,07 & 0,11 & 0,68 & 0,39 \\
\hline COPEPODES & & & & & & & & & 1,00 & 0,58 & 0,53 & 0,52 & $-0,21$ & 0,23 & 0,56 & 0,30 & 0,32 & $-0,07$ & $-0,32$ & $-0,11$ & $-0,19$ \\
\hline JUVENILES & & & & & & & & & & 1,00 & 0,84 & 0,43 & 0,19 & 0,05 & 0,17 & 0,46 & 0,29 & $-0,14$ & $-0,07$ & 0,21 & $-0,07$ \\
\hline TOTAL ZOOPLANCTON & & & & & & & & & & & 1,00 & 0,11 & 0,08 & 0,01 & 0,09 & 0,59 & $-0,03$ & 0,08 & 0,33 & $-0,17$ & $-0,08$ \\
\hline PHYTOCLASTES & & & & & & & & & & & & 1,00 & $-0,57$ & 0,06 & $-0,05$ & $-0,48$ & $-0,01$ & $-0,33$ & 0,14 & 0,60 & $-0,13$ \\
\hline ZOOCLASTES , & & & & & & & & & & & & & 1,00 & 0,12 & 0,46 & 0,58 & 0,66 & 0,25 & 0,44 & 0,20 & 0,31 \\
\hline MACROPHYTOCLASTES & & & & & & & & & & & & & & 1,00 & 0,41 & 0.11 & 0.53 & 0,66 & 0,58 & 0,15 & 0,83 \\
\hline PART. MINERALES & & & & & & & & & & & & & & & 1,00 & 0,44 & 0,73 & 0,34 & 0,07 & 0,40 & 0,19 \\
\hline AMT ELMT FIG & & & & & & & & & & & & & & & & 1,00 & 0,42 & 0,07 & 0.11 & $-0,45$ & $-0,08$ \\
\hline TOTAL ELMT FIGURES & & & & & & & & & & & & & & & & & 1,00 & 0,13 & 0,63 & 0,14 & 0,36 \\
\hline AGREGATS & & & & & & & & & & & & & & & & & & 1.00 & 0,09 & $-0,40$ & 0,83 \\
\hline FLOCONS & & & & & & & & & & & & & & & & & & & 1,00 & 0,54 & 0,55 \\
\hline MACROFLOCS & & & & & & & & & & & & & & & & & & & & 1,00 & 0,13 \\
\hline TOTAL ORG-MIN. & & & & & & & & & & & & & & & & & & & & & 100 \\
\hline
\end{tabular}

Dans chacun des 4 types statistiques définis cidessus, les variables sont associées entre elles par des valeurs de corrélations significatives au seuil de $5 \%$ et forment $\mathbf{4}$ groupes ou chaînes de corrélations clairement définies. Entre différentes variables de ces quatre types existent des corrélations de valeurs plus faibles, figurées en traits discontinus. L'existence de ces relations " intratypes fortes " et " intertypes moyennes ", suggère que ces quatre groupes de variables sont susceptibles de coexister dans l'écosystème lacustre, à condition qu'ils soient distincts dans l'espace ou dans le temps. La distance (spatiale ou temporelle) est d'autant plus grande que la corrélation négative entre deux groupes d'algues est forte, telle qu'entre Cyanophycées et Chrysophytes, Pyrrhophytes. Les fragments organiques planctoniques ( = bioclastes) assurent le lien entre les quatre groupes, et on peut penser que les bioclastes occupent dans l'écosystème lacustre une position charnière dans la succession temporelle des espèces, ou dans leur distribution verticale.

Parmi les particules organiques, les macrophytoclastes et les associations composites organominérales (agrégats, flocons, macroflocs) accompagnent les peuplements planctoniques à base de
Cyanophycées ou de Diatomées, algues montrant de fortes corrélations avec les concentrations de matière organique particulaire (Cyanophycées $r=+0,63$, Diatomées $r=+0,68$ ). Les autres particules organiques, telles que les phytoclastes, les zooclastes, ou les amoncellements d'éléments figurés; sont associées aux Chrysophytes, Pyrrhophytes et Chlorophytes, groupes d'algues qui entretiennent le zooplancton et qui ne présentent pas de corrélation significative avec la teneur en matière organique particulaire.

\subsubsection{Relations avec diverses composantes écologiques}

Si l'on confronte globalement les résultats obtenus en considérant les analyses lac par lac on se trouve, en ce qui concerne les échantillons analysés, devant trois cas de figure synthétisés au tableau 5.

* Dans le premier cas : la prolifération d'une ou de quelques espèces du phytoplancton engendre dans le seston une augmentation considérable du nombre de particules accompagnée par une contribution en masse supérieure à $3 \mathrm{mg} / \mathrm{l}$. C'est le cas des échantillons de Narlay et du Val. L'abondance du trypton peut être due, pour partie aux apports périphériques, 
et pour partie à l'intervention de bactéries minéralisatrices génératrices d'agrégats (Otsuki \& Hanya 1972). Les hypolimnions de ces lacs présentent les plus fortes désoxygénations enregistrées.

* Dans le second cas : le trypton est dominant et contribue, pour l'essentiel, à l'élévation de la charge pondérale en MES. Les hypolimnions sont, en anaérobiose prolongée, d'une intensité moyenne à forte (Rousses, Abbaye, Ilay).
* Dans le troisième cas : on ne trouve en excès ni phytoplancton, ni trypton. La concentration massique du seston est inférieure à $3 \mathrm{mg} / \mathrm{l}$ et la perte au feu inférieure à $1 \mathrm{mg} / \mathrm{l}$. C'est le cas des lacs de Clairvaux, St-Point, Maclu et Chalain dont les hypolimnions restent en aérobiose permanente (St-Point, Maclu) ou en anaérobiose modérée (Clairvaux, Chalain).

Tableau 5. Lien entre la nature, la concentration du seston et la désoxygénation des hypolimnions de 9 lacs du Jura français.

Table 5. Relationship between type and concentration of seston and oxygen depletion of hypolimnion from 9 lakes of the French Jura.

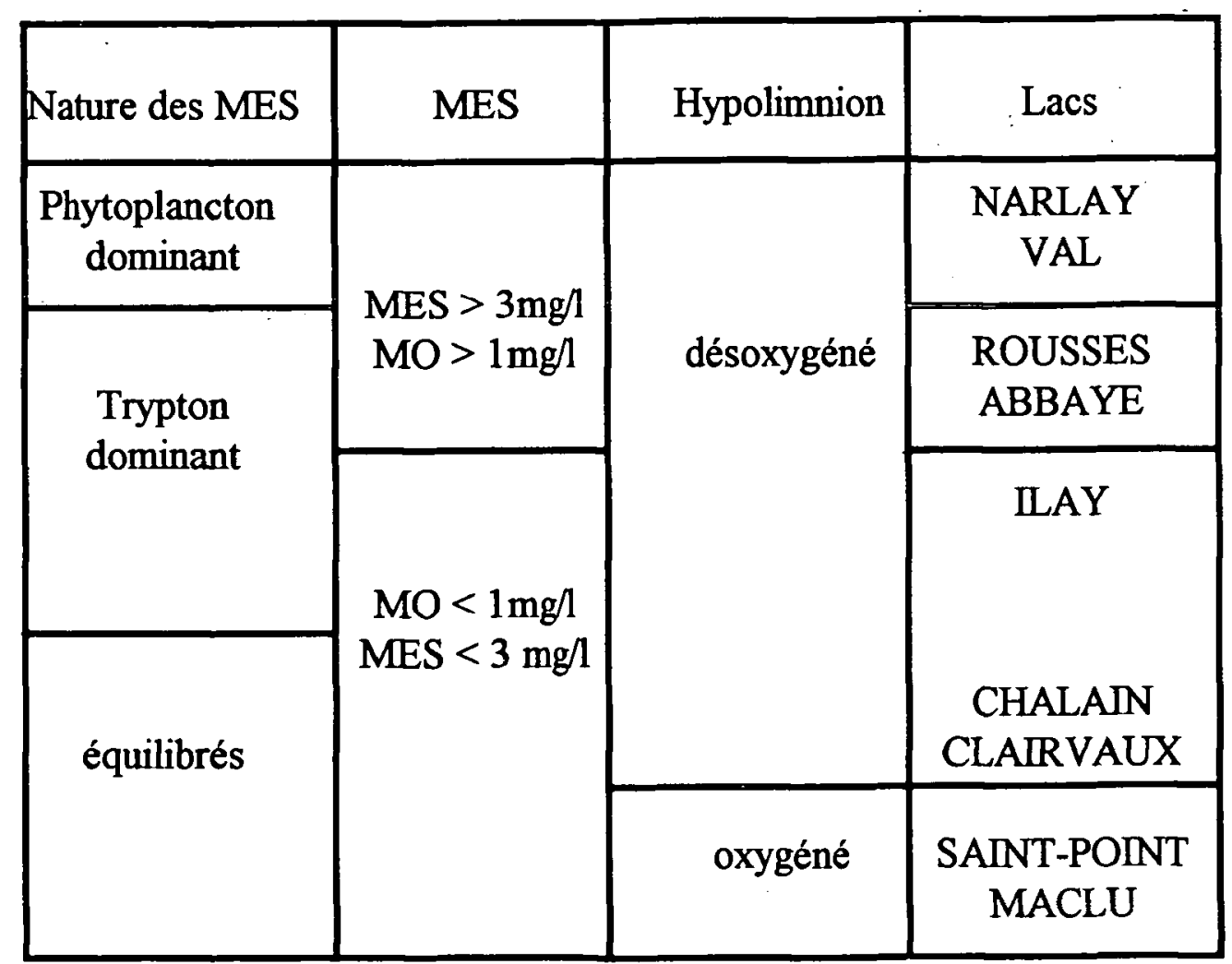

Tableau 6. Perte au feu (\% m.s.) dans les MES et les sédiments de 7 lacs jurassiens. (*) : d'après Verneaux et al. (1987). Table 6. Ignition loss (\% d.m.) in the Suspended Matter and Sediments of 7 lakes of the French Jura. $\left({ }^{*}\right):$ from Verneaux et al. (1987).

\begin{tabular}{lcc}
\hline$\%$ PF dans les MES & $30-50 \%$ & \\
$\%$ PF dans les sédiments $\left(^{*}\right)$ & $4-15 \%$ & $25-40 \%$ \\
\hline Lacs concernés & $\begin{array}{l}\text { Clairvaux - Chalain } \\
\text { Saint-Point - Maclu }\end{array}$ & $\begin{array}{c}\text { Rousses - Ilay } \\
\text { Abbaye }\end{array}$ \\
\hline
\end{tabular}


Par opposition au pourcentage de matière organique du seston qui varie dans une gamme étroite allant de 30 à $50 \%$, les valeurs rencontrées dans les sédiments ont une amplitude nettement plus importante (4 à $40 \%$, Tableau 6). Sur environ la moitié des échantillons on dose 3 fois plus de MO dans le seston que dans les sédiments, indiquant à l'instar de Mudroch (1984), de Stabel (1984, 1985), et de Stroffek (1990), une minéralisation primaire rapide et effective dans la colonne d'eau ou à l'interface eau-sédiment. Pour les autres, l'accumulation de matière organique résiduelle s'accompagne d'une désoxygénation de l'hypolimnion d'autant plus forte que la concentration en matière particulaire est élevée.

\section{Relations avec le mode de fonctionne- ment des écosystèmes lacustres}

L'analyse des abondances (Fig. 6 A, B, C) a montré que les associations organo-minérales composites, ainsi que les macrophytoclastes représentent la fraction majoritaire du trypton dans les lacs à péritrophie dominante (Rousses, Abbaye, Ilay) ou dans les lacs à forte production planctonique (Val, Narlay). Par opposition, les associations organominérales composites et le seston global (Fig .3) sont en quantité restreinte aux lacs pélagotrophes de Saint-Point, Maclu, Clairvaux et Chalain, en situation de lacs " chauds " en anaérobiose modérée (Clairvaux, Chalain), ou de lacs plus froids mais où le pourcentage de saturation en oxygène est supérieur à $20 \%$ quelle que soit l'époque ou la profondeur (Saint-Point, Maclu, Fig. 2).

L'importance de la frange littorale, et, sousendendu, la nature et la quantité de matière qui s'y rapporte, ainsi que les proliférations planctoniques apparaissent avec le couple oxygène/température comme les facteurs de la formation des associations organo-minérales. Cette interprétation, en accord avec les résultats expérimentaux de Godshalk \& Wetzel (1976) sur la décomposition des macrophytes, et les remarques de Wetzel (1983) et Verneaux et al. (1991) sur la nature des particules organiques, suggère que le mécanisme de la formation des associations organo-minérales composites serait essentiellement bactérien.

Lors de la sénescence et de la décomposition du phytoplancton, des algues macroscopiques ou des macrophytes aquatiques, une fraction majoritaire de composés organiques est libérée (30-70 \% du $\mathrm{COP}$ ) sous forme dissoute qui se trouve à l'origine de la croissance d'organismes hétérotrophes, (Saunders 1957, Hough \& Wetzel 1975, Robinson et al. 1982). La conversion du carbone organique dissous en carbone organique particulaire s'effectue alors sous l'action des bactéries hétérotrophes (Paerl 1974), qui créent un réseau favorable à la genèse d'associations organo-minérales composites (Simon \& Tilzer 1982) résultant de la production de polysaccharides bactériens extracellulaires jouant le rôle de glu biologique (Biddanda 1985, 1986).

Ainsi, de par leur genèse et les mécanismes de leur formation, les associations organo-minérales composites sont associées à des milieux dystrophes (Rousses, Abbaye) ou hypertrophes (Val, Narlay) et témoignent de la présence d'une intense activité bactérienne de décomposition.

Dans le diagramme des corrélations interparamétriques (Fig. 7), réalisé tous échantillons confondus, les Cyanophycées et les Diatomées sont corrélées, avec les associations composites néoformées, et les Chrysophytes, Pyrrhophytes, Chlorophytes avec les organismes consommateurs secondaires. Dans ce schéma, les bioclastes (zooclastes, phytoclastes), occupent une position charnière dans les successions temporelles, ou dans la distribution spatiale des types statistiques identifiés par l'analyse. A l'opposé, les macrophytoclastes se corrèlent fortement aux associations organo-minérales du type flocons et agrégats, ainsi qu'aux Cyanophycées et Diatomées.

Ces observations permettent de proposer une conception au devenir de la MOP lacustre dans laquelle les associations organo-minérales composites, produits secondaires de la minéralisation bactérienne des produits frais, constituent des formes de blocage de la MOP assimilables à des impasses trophiques, et qui se juxtaposent au transfert de la MOP vers les échelons de consommation supérieurs.

\section{Conclusions}

a) Le trypton représente en moyenne $30 \%$ du nombre des particules en suspension ;

b) Deux types principaux de particules détritiques sont observés :

- les détritus de Wetzel (1983) qui sont des fragments organiques provenant principalement de la fragmentation ou de la dilacération de tissus végétaux ou d'organismes planctoniques, 
- les recombinaisons de matière organique dissoute ou particulaire pour la formation d'associations organo-minérales composites ;

c) L'abondance des particules organiques détritiques et notamment des associations organominérales composites, est maximale dans les systèmes désoxygénés ;

d) Trois facteurs environnementaux de la formation des associations organo-minérales sont évoqués : l'importance de la frange littorale, le couple , oxygène/température, les proliférations planctoniques ;

e) L'étude des corrélations interparamétriques montre la juxtaposition dans l'écosystème lacustre, de quatre types statistiques d'associations particulaires, parmi lesquelles s'opposent les particules organiques néoformées (forme de blocage de la MOP), et les organismes consommateurs secondaires (forme de transfert de la MOP).

Malgré l'activité bactérienne susceptible de minéraliser de 70 à $85 \%$ du carbone organique dans la colonne d'eau (Fallon \& Brock 1980, Stroffek 1990), la notion d'abondance développée dans l'analyse " in toto " de la matière en suspension de 9 lacs jurassiens, montre que la décomposition bactérienne ne permet pas de résorber l'intégralité des apports organiques dont une fraction particulaire (macrophytoclastes, zooclastes) s'accumule au niveau du sédiment (Verneaux et al. 1991), et une fraction remaniée (associations organo-minérales composites), produit secondaire de la minéralisation bactérienne, séjourne dans la colonne d'eau. On peut raisonnablement estimer qu'un transfert optimal de la MOP serait plutôt caractérisé par la multiplication des voies de transfert de l'énergie vers les échelons de consommation supérieurs (chaîne prédatrice), impliquant la limitation optimale des substances organiques résiduelles.

Les recherches actuelles s'appliquent, à préciser la nature des associations organo-minérales, à vérifier les hypothèses de concordance émises précédemment, et abordent quantitativement la sédimentation particulaire dans le lac dysfonctionnel le plus représentatif de notre échantillon, le lac d'Ilay.

\section{Remerciements}

Je remercie M. Vidonne, pour sa disponibilité et son assistance, au cours des analyses chimiques, $\mathbf{M}$. Gomot et Mme Bride qui m'ont réservé le meilleur accueil au Laboratoire de Zoologie, lors des nombreuses séances de photo-microscopie, et M. Guyard pour ses lectures attentives et ses critiques. Mes remerciements s'adressent particulièrement à $\mathrm{M}$. Verneaux pour l'intérêt porté à ce travail et les remarques relatives au manuscrit. Les suggestions et les commentaires apportés par M. Golterman et M. Meybeck, m'ont été d'une aide précieuse pour aboutir à la version définitive de ce texte, qu'ils trouvent ici l'expression de ma reconnaissance.

\section{Travaux cités}

Biddanda B.A. 1985. - Microbial synthesis of macroparticulate matter. Mar. Ecol. Prog. Ser. $20: 241-251$.

Biddanda B.A. 1986. - Structure and function of marine microbial aggregates. Oceanologica acta. 9 (2) : 209-211.

Blanc F. 1968. - Etude comparée de quelques méthodes d'estimation quantitative et qualitative du matériel particulaire en suspension dans l'eau de mer. Thèse Doct. Océanogr. Marseille, $72 \mathrm{p}$.

Bloesch J. 1974. - Sedimentation und Phosphorhaushalt im Vierwaldstattersee (Horwer Bucht) und im Rotsee. Schweitz. Z. Hydrol. 36 (1) : 71-186.

Bloesch J., Stadelmann P. \& Bührer H. 1977. - Primary production, mineralization, and sedimentation in the euphotic zone of two Swiss Lakes. Limnol. Oceanogr. 22 (3) : 511-526.

Bourrelly P. 1972. - Les Algues d'eau douce. Tome I : Les Algues vertes. 2nd éd. : N. Boubée et Cie, Paris, 569 p.

Campy M. 1982. - Le Quaternaire franc-comtois. Essai chronologique et paléoclimatique. Th. Univ. Fr-Comté, 575 p.

. Dussart B. 1966. - Limnologie : l'étude des eaux continentales. Gauthier-Villars Ed., 675 p.

Elliott J.M. \& Décamps H. 1973. - Guide pour l'analyse statistique des échantillons d'invertébrés benthiques. Annls Limnol. 9 (2) : 79-120.

Fallon R.D. \& Brock T.D. 1980. - Planktonic blue-green algae : Production, sedimentation, and decomposition in Lake Mendota, Wisconsin. Limnol. Oceanogr. 25 (1) : 72-88.

Gandais V. 1989. - Origines et variations spatio-temporelles des flux de matière particulaire au centre du Léman. Mém. Thèse Univ. Genève, $\mathrm{n}^{\circ} 2345,104 \mathrm{p}$.

Godshalk G.L. \& Wetzel R.G. 1976. - Decomposition of macrophytes and the metabolism of organic matter in sediments. In : H.L. Golterman (ed) : Interaction between sediments and freshwater : 258-264. Proc. SIL-UNESCO Symp. Sept 6-10, Amsterdam, Pudoc-Junk, Wageningen.

Golterman H.L., Sly P.G. \& Thomas R.L. 1983. - Study of the relationship between water quality and sediment transport. Ed. UNESCO, Paris, 231 p.

Gordon D.C. 1970. - A microscopic study of organic particles in the North Atlantic Ocean. Deep-Sea Research, 17 : 175-185.

Hölemann J. \& Wirth H. 1988. - Concentration, Major Element Ratios and Scanning Electron Microscopy of Suspended Particulate Matter from the North Sea, Spring 1986. Mitt. Geol.-Paläont. Inst. Univ. Hamburg, Heft 65 : 183-206.

Hough R.A. \& Wetzel R.G. 1975. - The release of dissolved organic carbon from submersed aquatic macrophytes : Diel, seasonal, and community relationships. Verh. Internat. Verein. Limnol., 19 : 939-948.

Hunt J.R. 1982. - Particle Dynamics in Seawater : implications for Predicting the Fate of Discharged Particles. Environ. Sci. Technol. 16 (6) : 303-309. 
Kelts K. \& Hsu K.J. 1978. - Freshwater Carbonate Sedimentation. In : A. Lerman ed. Lakes, Chemistry, Geology, Physics. Springer Verlag, New-York, 295-323.

Küchler-Krischun J. \& Kleiner J. 1990. - Heterogeneously nucleated calcite precipitation in Lake Constance. A short time resolution study. Aquatic sciences, 52 (2) : 176-197.

Landmann G. 1988. - An In Situ Camera System, Developed and Tesied for the Characterization of Suspended Matter in the Ocean. Mitt. Geol.-Paläont. Inst. Univ. Hamburg, Heft $65: 207-228$.

Lund J.W.G.. Kipling C. \& Le Cren E.D. 1958. - The inverted microscope method of estimating algal numbers and the statistical basis of estimations by counting. Hydrobiologia, 11 : 143-170.

Manheim F.T., Hathaway J.C. \& Uchupi E. 1972. - Suspended matter in surface waters of the northern Gulf of Mexico. Limnol. Oceanogr., V, 17 (1) : 17-27.

Mudroch A. 1984. - Chemistry, mineralogy, and morphology of Lake Erie Suspended Matter. J. Great Lakes Res. 10 (3) : 286-298.

Otsuki A. \& Hanya T. 1972. - Production of dissolved organic matter from dead green algal cells. 1. Aerobic microbial decomposition. Limnol. Oceanogr., 17 (2) : 248-257. 2. Anaerobic microbial decomposition. Limnol. Oceanogr., 17 (2) : 258-264.

Paerl H.W. 1974. - - Bacterial uptake of dissolved organic matter in relation to detrital aggregation in marine and freshwater systems. Limnol. Oceanogr., V 19 (6) : 966-972.

Riley G.A. 1963. - Organic aggregates in seawater and the dynamics of their formation and utilization. Limnol. Oceanogr., $8: 372-381$.

Robinson J.D., Mann K.H. \& Novitsky J.A. 1982. -Conversion of the particulate fraction of seaweed detritus to bacterial biomass. Limnol. Oceanogr., 27 (6) : 1072-1079.

Saunders G.W. 1957. - Interrelations of dissolved organic matter and phytoplankton. Botanical Review, 23 (8-9) : 389-409.
Simon M. \& Tilzer M.M. 1982. - Bacterial decay of the autumnal phytoplankton in Lake Constance Bodensee. Schweiz. $z$. Hydrol. 44/2 : 263-275.

Stabel H.-H. 1984. - Impact of sedimentation on the phosphorus content of the euphotic zone of Lake Constance. Verh. Internat. Verein Limnol., 22 : 964-969.

Stabel H.-H. 1985. - Mechanisms controlling the sedimentation sequence of various elements in prealpine lakes. In W. Stumm : Chemical processes in Lakes. Ed. J. Wiley, NewYork, $435 \mathrm{p}$.

Stabel H.-H. 1986. - Calcite precipitation in Lake Constance : Chemical equilibrium, sedimentation, and nucleation by algae. Limnol. Oceanogr., 31 (5) : 1081-1093.

Stroffek S. 1990. - Les transferts verticaux de matière et leur modification par les bactéries hétérotrophes fixées sur les particules en sédimentation dans les eaux de surface de deux grands lacs alpins (lac Léman, lac du Bourget, France). Mém. Thèse Univ. Lyon $\mathrm{I}^{\circ} 22190,175 \mathrm{p}$.

Sturm M. 1985. - Schwebstoffe in Seen. Particules en suspension dans les lacs. Mitt/Nouv. EAWAG 19 : 9-15.

Sugiura T. 1981: - Organic composition of particulate mater sinking in lake water. Verh. Internat. Verein. Limnol., 21 : 624-627.

Verneaux J., Remy F., Vidonne A. \& Guyard A. 1987. Caractères généraux des sédiments de 10 lacs jurassiens. Sci. Eau 6 : 107-128.

Verneaux J., Vidonne A., Remy F. \& Guyard A. 1991. Particules organiques et rapport $\mathrm{C} / \mathrm{N}$ des sédiments des lacs du Jura. Annls Limnol., 27 (2) : 175-190.

Wetzel R.G. 1983. - Limnology. 2nd éd. : CBS College Publishing, New-York, Philadelphia, 767 p + annexes.

Wirth H. \& Seifert R. 1988. - Mineralogy, Geochemistry and SEM-Observations of Suspended Particulate Matter from the North Sea, Spring 1984. Mitt. Geol. Paläont. Inst. Univ. Hamburg, Heft $65: 163-181$.

Zumstein J. \& Buffle J. 1989. - Circulation of pedogenic and aquagenic organic matter in an eutrophic lake. Wat. Res., 23 (2) : 229-239. 